\title{
Quantum Ising Hamiltonian Programming in Trio, Quartet, and Sextet Qubit Systems
}

\author{
Minhyuk Kim®, ${ }^{1}$ Yunheung Song $\odot,{ }^{1}$ Jaewan Kim $\odot,{ }^{2}$ and Jaewook Ahn $\oplus^{1, *}$ \\ ${ }^{1}$ Department of Physics, KAIST, Daejeon 34141, Korea \\ ${ }^{2}$ Department of Physics, Myongii University, Yongin 17058, Korea
}

(Received 8 September 2020; accepted 4 December 2020; published 18 December 2020)

\begin{abstract}
Rydberg-atom quantum simulators are of keen interest because of their possibilities towards highdimensional qubit architectures. Here we report continuous tuning of quantum Ising Hamiltonians of Rydberg atoms in three-dimensional arrangements. Various connected graphs of Rydberg atoms constructed with vertices and edges respectively representing atoms and Rydberg-blockaded atom pairs, and their eigenenergies are probed along with their geometric intermediates during structural transformations. Conformation spectra of star, complete, cyclic, and diamond graphs are probed for four interacting atoms and antiprism structures for six atoms. The energy level shifts and merges of the tested structural transformations are clearly observed with Fourier-transform spectroscopy, in good agreement with the model few-body quantum Ising Hamiltonian. This result demonstrates the possibility of continuous geometry tuning and thus programming of many-body spin-Hamiltonian systems.
\end{abstract}

DOI: 10.1103/PRXQuantum.1.020323

\section{INTRODUCTION}

Well-calibrated quantum many-body systems are currently in high demand because of their essential necessities for quantum applications such as quantum computing and quantum simulation [1-3]. Among many promising physical platforms [4-8], Rydberg-atom quantum simulators, which use a mesoscopic-scale, deterministic arrangement of neutral atoms with controllable strong local interactions induced by Rydberg-atom excitation, draw latest attentions [9]. They have well-defined energy levels and relatively long coherence and lifetimes, and entanglements in these systems are generated with relative ease through giant dipole-dipole couplings in the Rydberg-atom blockade regime [10-12]. In recent demonstrations, these systems are used to generate as many as 20-qubit GreenbergerHorne-Zeilinger entangled states [13], to observe the quantum many-body phenomena involved with localization [14] and thermalization [15], and also to investigate the critical phenomena of Ising-type or $X Y$ quantum spin models across phase transitions [16-18]. Of particular importance in the context relevant to the present work,

*jwahn@kaist.ac.kr

Published by the American Physical Society under the terms of the Creative Commons Attribution 4.0 International license. Further distribution of this work must maintain attribution to the author(s) and the published article's title, journal citation, and DOI.
Rydberg-atom quantum simulators are expected to realize the possibilities of high-dimensional qubit architectures [19-23].

Quantum simulation uses a controllable Hamiltonian $H$ of a quantum $N$-body system to reproduce or predict the behavior of other equivalent systems of model Hamiltonian $H_{G}$. The system size $N$ increases computational power exponentially, and the controllability in $H$ increases the simulation accuracy $[24,25]$ as well as the diversity of the systems to be simulated. Currently, Rydberg-atom quantum simulators have scaled up the number of qubits [26], approaching the regime of quantum supremacy [27]. In that regard, dexterous control of the parameters of $H$, as many as possible and with high fidelities, is of great importance for a programmable quantum simulator. In this paper, we use a Rydberg-atom quantum simulator, of Hamiltonian $H$, to produce and tune a set of twoor three-dimensional arrangements of atoms, which are isomorphic to connected graphs $\{G\}$ of $N=3-6$ qubits, to model the quantum Ising Hamiltonian $H_{G}$, and probe their graph-dependent eigenspectra, in particular, during structural transformations from one $G$ to another.

\section{QUANTUM ISING HAMILTONIAN}

We model the Hamiltonian $H$ of a Rydberg-atom quantum simulator to the quantum Ising Hamiltonian $H_{G}$ for a connected graph $G(V, E)$, where the vertices $V$ denote atoms and the edges $E$ denote nearest-neighbor atom pairs, as follows. The Hamiltonian of $N$ atoms that are coherently 
excited to a Rydberg energy state is given by

$$
\hat{H}=\frac{\hbar \Omega}{2} \sum_{j=1}^{N}\left(|1\rangle_{j}\left\langle\left. 0\right|_{j}+\mid 0\right\rangle_{j}\left\langle\left. 1\right|_{j}\right)+\sum_{j<k} U\left(r_{j k}\right) \hat{n}_{j} \hat{n}_{k},\right.
$$

where $|0\rangle_{j}$ and $|1\rangle_{j}$ denote the ground and Rydberg energy states, respectively, of an atom $j$ located at $\vec{r}_{j}, \Omega$ is the Rabi oscillation frequency, $U\left(r_{j k}\right)=C_{6} /\left|\vec{r}_{j}-\vec{r}_{k}\right|^{6}$ is the van der Waals interaction between two Rydberg atoms, and $\hat{n}_{j}=|1\rangle_{j}\left\langle\left. 1\right|_{j}\right.$ is the excitation number [10]. In the following, we consider arrangements of atoms (e.g., see Fig. 1) in which only the nearest-neighbor pairs, of the same interatom distance $d$, are within the Rydberg-blockade radius, i.e., $d<r_{b}=\left|C_{6} / \hbar \Omega\right|^{1 / 6}$, and no other atom pairs are. Such an arrangement of atoms can be isomorphically represented by an undirected connected graph $G(V, E)$, if the interactions of other pairs are ignorable [28]. Then, the Hamiltonian $H$ for a graph $G$ is the quantum Ising Hamiltonian $H_{G}$ (with inhomogeneous longitudinal field), given by

$$
\hat{H}_{G}=J \sum_{(j, k) \in E} \hat{\sigma}_{z}^{(j)} \hat{\sigma}_{z}^{(k)}+\sum_{j}^{N}\left(h_{x} \hat{\sigma}_{x}^{(j)}+h_{z}^{(j)} \hat{\sigma}_{z}^{(j)}\right),
$$

in which $\hat{\sigma}_{x, z}$ are Pauli spins, $J=U(d) / 4$ is the exchange interaction, $h_{x}=\hbar \Omega / 2$ is the transverse field, and $h_{z}^{(j)}=$ $-\left\|E_{j}\right\| U(d) / 4$ is the longitudinal field with $\left\|E_{j}\right\|$ the number of edges for vertex $j$.

In order to investigate the structural change of a strongly coupled Rydberg-atom system, we consider, for example, four-atom $(N=4)$ arrangements and their structural transformations. As shown in Fig. 1(a), there are four nonisomorphic 4-vertex-connected graphs: the star graph, denoted by $S_{4}$, has one atom at the center and three at the ends of three claws, and three nearest-neighbor edges $(E=$ $3)$; the complete graph, $K_{4}$, has four atoms in the tetrahedron configuration with six edges $(E=6)$; the cyclic graph, $C_{4}$, is the square configuration with four edges $(E=$ $4)$, and the diamond graph, $K_{4}-e$, has five edges $(E=5)$. Here, $S_{4}, C_{4}$, and $K_{4}-e$ are two dimensional (e.g., in the $x-y$ plane) and $K_{4}$ is three dimensional. Structural transformations from one atom arrangement to another follow, for example, through the sequences $S_{4} \rightarrow K_{4}, K_{4} \rightarrow C_{4}$, and $C_{4} \rightarrow K_{4}-e$, as respectively shown in Figs. 1(b)-1(d).

As a pedagogical example, let us consider $C_{4}$, the cyclic graph, which has four nearest-neighbor couplings. Because of the symmetry of the given graph, most of the possible $2^{N}=16$ eigenstates of $H_{G}$ are dark states, inaccessible from the initial state $|\Psi(t=0)\rangle=|0000\rangle$, the bare-atom ground state, in our consideration. There are three bright eigenstates, of respective energies (in $\hbar=1$ unit hereafter) $\lambda_{1}=-\sqrt{\frac{3}{2}} \Omega, \lambda_{5}=0$, and $\lambda_{7}=\sqrt{\frac{3}{2}} \Omega$, where the index $j$ (a)
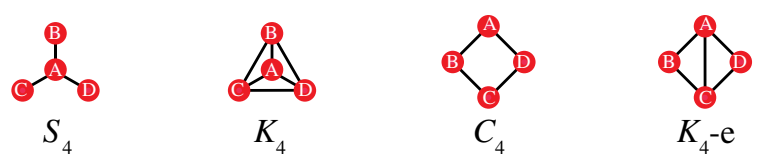

(b) $S_{4} \rightarrow K_{4}$

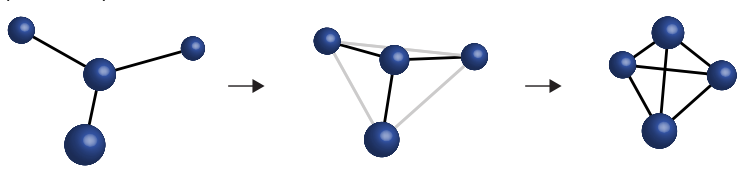

(c)

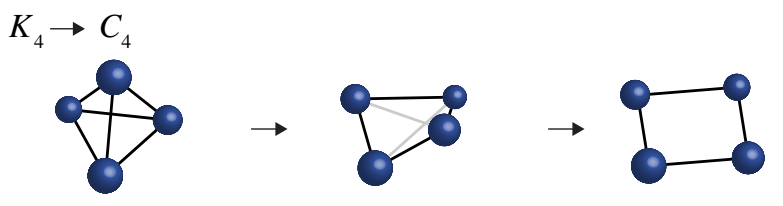

(d) $\mathrm{C}_{4} \rightarrow K_{4}-\mathrm{e}$

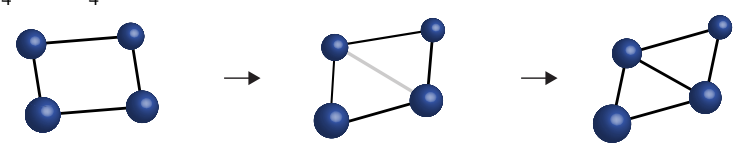

FIG. 1. (a) Connected graphs for $N=4$ atom arrangements: $S_{4}$, the star graph, $K_{4}$, the complete graph, $C_{4}$, the cycle graph, and $K_{4}-e$, the diamond graph, in which vertices and edges represent atoms and Rydberg-blockaded atom pairs, respectively. (b)-(d) Structural changes from (b) $S_{4}$ to $K_{4}$, (c) $K_{4}$ to $C_{4}$, (d) $\mathrm{C}_{4}$ to $\mathrm{K}_{4}-e$.

denotes the energy ordering among all energy states, bright and dark. The corresponding eigenstates are given by

$$
\begin{aligned}
& \left|\lambda_{1}\right\rangle=-\frac{1}{\sqrt{3}}\left|W_{0}\right\rangle+\frac{1}{\sqrt{2}}\left|W_{1}\right\rangle-\frac{1}{\sqrt{6}}\left|W_{2}^{C}\right\rangle, \\
& \left|\lambda_{5}\right\rangle=-\frac{1}{\sqrt{3}}\left|W_{0}\right\rangle+\sqrt{\frac{2}{3}}\left|W_{2}^{C}\right\rangle, \\
& \left|\lambda_{7}\right\rangle=\frac{1}{\sqrt{3}}\left|W_{0}\right\rangle+\frac{1}{\sqrt{2}}\left|W_{1}\right\rangle+\frac{1}{\sqrt{6}}\left|W_{2}^{C}\right\rangle,
\end{aligned}
$$

which are represented with symmetric base states, $\left|W_{0}\right\rangle=$ $|0000\rangle,\left|W_{1}\right\rangle=(|1000\rangle+|0100\rangle+|0010\rangle+|0001\rangle) / \sqrt{4}$, and $\left|W_{2}^{C}\right\rangle=(|1010\rangle+|0101\rangle) / \sqrt{2}$, each labeled with an excitation number (the number of atoms in the Rydberg state). Likewise, there are four bright eigenstates for $S_{4}$, two for $K_{4}$, and four for $K_{4}-e$. In Table I we summarize the eigenenergies and eigenstates of $H_{G}$ for the four graphs. Note that the eigenenergies of $H_{G}$ could differ from $H$ by up to an order of the next-nearest-neighbor interaction, $U(d) / 8$ in $C_{4}$ or $U(d) / 27$ in $S_{4}$.

\section{RYDBERG-ATOM QUANTUM SIMULATOR}

In order to probe the eigenspectra of an $N$-body quantum Ising Hamiltonian, we use a Rydberg-atom quantum simulator, which can (i) arrange $N$ single atoms isomorphically to a connected graph, (ii) implement the Hamiltonian $H$ 
TABLE I. Quantum Ising eigenstates of $N=4$ atom systems (bright states only), in symmetric base states defined by $\left|W_{0}\right\rangle=|0000\rangle$, $\left|W_{1}\right\rangle=(|1000\rangle+|0100\rangle+|0010\rangle+|0001\rangle) / \sqrt{4},\left|W_{2}^{C}\right\rangle=(|1010\rangle+|0101\rangle) / \sqrt{2},\left|W_{1}^{S}\right\rangle=(|0100\rangle+|0010\rangle+|0001\rangle) / \sqrt{3},\left|W_{2}^{S}\right\rangle=$ $(|0110\rangle+|0011\rangle+|0101\rangle) / \sqrt{3},\left|W_{1}^{D}\right\rangle=(|1000\rangle+|0010\rangle) / \sqrt{2}$, and $\left|W_{1}^{D}\right\rangle=(|0100\rangle+|0001\rangle) / \sqrt{2}$.

\begin{tabular}{|c|c|c|}
\hline Configuration & Eigenenergies $(\hbar=1)$ & Eigenstates (bright states only) \\
\hline Star graph $S_{4}$ & $\begin{array}{l}\lambda_{1}=-\sqrt{\frac{23}{10}} \Omega \\
\lambda_{2}=-\sqrt{\frac{10}{23}} \Omega \\
\lambda_{8}=\sqrt{\frac{10}{23}} \Omega \\
\lambda_{9}=\sqrt{\frac{23}{10}} \Omega\end{array}$ & $\begin{array}{l}\left|\lambda_{1}\right\rangle=\sqrt{\frac{3}{20}}\left|W_{0}\right\rangle-\sqrt{\frac{11}{30}}\left|W_{1}^{S}\right\rangle-\sqrt{\frac{1}{60}}|1000\rangle+\sqrt{\frac{7}{20}}\left|W_{2}^{S}\right\rangle-\sqrt{\frac{7}{60}}|0111\rangle \\
\left|\lambda_{2}\right\rangle=-\sqrt{\frac{7}{20}}\left|W_{0}\right\rangle+\sqrt{\frac{1}{30}}\left|W_{1}^{S}\right\rangle+\sqrt{\frac{1}{5}}|1000\rangle+\sqrt{\frac{3}{20}}\left|W_{2}^{S}\right\rangle-\sqrt{\frac{4}{15}}|0111\rangle \\
\left|\lambda_{8}\right\rangle=\sqrt{\frac{7}{20}}\left|W_{0}\right\rangle+\sqrt{\frac{1}{30}}\left|W_{1}^{S}\right\rangle+\sqrt{\frac{1}{5}}|1000\rangle-\sqrt{\frac{3}{20}}\left|W_{2}^{S}\right\rangle-\sqrt{\frac{4}{15}}|0111\rangle \\
\left|\lambda_{9}\right\rangle=\sqrt{\frac{3}{20}}\left|W_{0}\right\rangle+\sqrt{\frac{11}{30}}\left|W_{1}^{S}\right\rangle+\sqrt{\frac{1}{60}}|1000\rangle+\sqrt{\frac{7}{20}}\left|W_{2}^{S}\right\rangle+\sqrt{\frac{7}{60}}|0111\rangle\end{array}$ \\
\hline Complete graph $K_{4}$ & $\begin{array}{l}\lambda_{1}=-\Omega \\
\lambda_{5}=\Omega\end{array}$ & $\begin{aligned}\left|\lambda_{1}\right\rangle & =\sqrt{\frac{1}{2}}\left|W_{0}\right\rangle-\sqrt{\frac{1}{2}}\left|W_{1}\right\rangle \\
\left|\lambda_{5}\right\rangle & =\sqrt{\frac{1}{2}}\left|W_{0}\right\rangle+\sqrt{\frac{1}{2}}\left|W_{1}\right\rangle\end{aligned}$ \\
\hline Cyclic graph $C_{4}$ & $\begin{array}{l}\lambda_{1}=-\sqrt{\frac{3}{2}} \Omega \\
\lambda_{5}=0 \\
\lambda_{7}=\sqrt{\frac{3}{2}} \Omega\end{array}$ & $\begin{aligned}\left|\lambda_{1}\right\rangle & =-\sqrt{\frac{1}{3}}\left|W_{0}\right\rangle+\sqrt{\frac{1}{2}}\left|W_{1}\right\rangle-\sqrt{\frac{1}{6}}\left|W_{2}^{C}\right\rangle \\
\left|\lambda_{5}\right\rangle & =-\sqrt{\frac{1}{3}}\left|W_{0}\right\rangle+\sqrt{\frac{2}{3}}\left|W_{2}^{C}\right\rangle \\
\left|\lambda_{7}\right\rangle & =\sqrt{\frac{1}{3}}\left|W_{0}\right\rangle+\sqrt{\frac{1}{2}}\left|W_{1}\right\rangle+\sqrt{\frac{1}{6}}\left|W_{2}^{C}\right\rangle\end{aligned}$ \\
\hline Diamond graph $K_{4}-e$ & $\begin{array}{l}\lambda_{1}=-\sqrt{\frac{13}{10}} \Omega \\
\lambda_{2}=-\sqrt{\frac{5}{26}} \Omega \\
\lambda_{5}=\sqrt{\frac{5}{26}} \Omega \\
\lambda_{6}=\sqrt{\frac{23}{10}} \Omega\end{array}$ & $\begin{array}{l}\left|\lambda_{1}\right\rangle=-\frac{3}{5}\left|W_{0}\right\rangle+\frac{3}{5}\left|W_{1}^{D}\right\rangle+\sqrt{\frac{7}{50}}\left|W_{1}^{D}\right\rangle-\sqrt{\frac{7}{50}}|1010\rangle \\
\left|\lambda_{2}\right\rangle=-\sqrt{\frac{7}{50}}\left|W_{0}\right\rangle-\sqrt{\frac{7}{50}}\left|W_{1}^{D}\right\rangle+\frac{3}{5}\left|W_{1}^{D}\right\rangle+\frac{3}{5}|1010\rangle \\
\left|\lambda_{5}\right\rangle=\sqrt{\frac{7}{50}}\left|W_{0}\right\rangle-\sqrt{\frac{7}{50}}\left|W_{1}^{D}\right\rangle+\frac{3}{5}\left|W_{1}^{D}\right\rangle-\frac{3}{5}|1010\rangle \\
\left|\lambda_{6}\right\rangle=\frac{3}{5}\left|W_{0}\right\rangle+\frac{3}{5}\left|W_{1}^{D}\right\rangle+\sqrt{\frac{7}{50}}\left|W_{1}^{D}\right\rangle+\sqrt{\frac{7}{50}}|1010\rangle\end{array}$ \\
\hline
\end{tabular}

in Eq. (1) by creating Rydberg atoms, and (iii) readout the final state $|\Psi(t)\rangle$ after time $t$. We use the probability $P_{0}(t)$ of all atoms back to the initial state, defined by

$$
P_{0}(t)=\left|\left\langle W_{0} \mid \Psi(t)\right\rangle\right|^{2}=\left|\left\langle W_{0}\left|e^{-i \hat{H} t / \hbar}\right| W_{0}\right\rangle\right|^{2},
$$

where $\left|W_{0}\right\rangle$ is the initial zero-excitation state, e.g., $\left|W_{0}\right\rangle=$ $|000\rangle$ for $N=3,|0000\rangle$ for $N=4$, and $|000000\rangle$ for $N=$ 6. With eigenenergies $\lambda_{j}, P_{0}(t)$ is given by

$$
P_{0}(t)=\sum_{j}\left|A_{j}\right|^{4}+\sum_{j<k} B_{j k} \cos \left(\lambda_{j k} t\right)
$$

with $A_{j}=\left\langle W_{0} \mid \lambda_{j}\right\rangle$ and $B_{j k}=2\left|A_{j}\right|^{2}\left|A_{k}\right|^{2}$. So the Fourier transform, $\mathcal{F}\left[P_{0}(t)\right]$, retrieves the energy differences $\lambda_{j k}=$ $\lambda_{j}-\lambda_{k}$ for all pairs of eigenenergies.

Experiments are performed with an updated version of the machine previously used in our earlier works for deterministic multiatom arrangements [21,29,30], quantum many-body thermalization [15], and entanglement generations [31]. For the current work, we have increased the Rabi coherence time [32] to about $10 \mu \mathrm{s}$, and have developed a new method of three-dimensional atom arrangements; see Sec. V. The procedures are summarized as follows.

(i) Atom arrangement. Single atoms are trapped with optical tweezers and arranged in three-dimensional space. Rubidium $\left({ }^{87} \mathrm{Rb}\right)$ atoms are first cooled to below $30 \mu \mathrm{K}$ by
Doppler and polarization gradient cooling in a magnetooptical trap, and optically pumped to the ground hyperfine state $|0\rangle=\left|5 S_{1 / 2}, F=2, m_{F}=2\right\rangle$. Then, a spatial light modulator (Meadowlarks $512 \times 512 \mathrm{XY}$ modulator) turns on as many as 250 optical tweezers (off-resonant optical dipole traps) to capture and rearrange $N$ single atoms deterministically to target positions, with $5-10 \mu \mathrm{m}$ spacing $[30,35]$. The wavelength of the optical tweezers is $820 \mathrm{~nm}$ and an objective lens (Mitutoyo G Plan Apo $50 \times$ ) of a high numerical aperture $(\mathrm{NA}=0.5)$ is used. The trap depth and diameter are $1 \mathrm{mK}$ and $2 \mu \mathrm{m}$, respectively, and the lifetime of each trapped atom is about $40 \mathrm{~s}$.

(ii) Implementation of $H$. With the optical tweezers turned off, the Hamiltonian $H$ in Eq. (1) is implemented, through the Rydberg-atom two-photon excitation from $|0\rangle$ to $|1\rangle=\left|71 S_{1 / 2}, m_{J}=\frac{1}{2}\right\rangle$ via the off-resonant intermediate state $|m\rangle=\left|5 P_{3 / 2}, F^{\prime}=3, m_{F}^{\prime}=3\right\rangle$. For the two-photon excitation, we use 780-nm (Toptica DL Pro) and 480-nm (Toptica TA-SHG Pro) lasers, of which the beams counterpropagate with diameters of about 180 and $35 \mu \mathrm{m}$, respectively, and the laser frequencies are stabilized to a linewidth less than $(2 \pi) 30 \mathrm{kHz}$, with a reference cavity (from Stable Laser Systems of finesse 15000). The Rabi frequency of the given two-photon transition is $\Omega=\Omega_{0 m} \Omega_{m 1} /\left(2 \Delta_{0 m}\right)$, when the intermediate detuning is $\Delta_{0 m}=-(2 \pi) 600 \mathrm{MHz}$. Measured Rabi frequencies, calibrated with reference single atoms, are $\Omega=(2 \pi) 1.0(1)$ $\mathrm{MHz}$ for $N=3,4$ experiments in Sec. IV and $(2 \pi) 0.8(1)$ $\mathrm{MHz}$ for the $N=6$ experiment. Correspondingly, the 
Rydberg blockade radii are given by $r_{b}=\left|C_{6} / \hbar \Omega\right|^{1 / 6}=$ 10(1) and 11(1) $\mu \mathrm{m}$, respectively.

(iii) Final state readout. In the detection stage, the optical tweezers are turned back on and the fluorescence from trapped ground-state atoms in $|0\rangle$ is collected through the same objective lens and imaged onto an electron multiplying charge-coupled device (EMCCD) camera. The tomographic images of a three-dimensional atomic array structure are obtained with an electrically focus-tunable lens (ETL, EL-16-40-TC from Optotune), located after the tube lens, by sequentially shifting the focal length [19]. At the same time, the EMCCD is triggered on and off with a period of $40 \mathrm{~ms}$ (camera exposure time) for each tomogram. After interaction $H$ of duration $t$, up to $5 \mu \mathrm{s}$ with time step $\Delta t=0.1 \mu \mathrm{s}$, the events of all atoms back in the bare-atom ground states are collected, and procedures (i)-(iii) are repeated about 100-200 times of data accumulation to obtain the $\left|W_{0}\right\rangle$-state probability in Eq. (5).

\section{RESULTS AND ANALYSIS}

To begin with a simplest possible deformation, we consider a three-atom configuration, which changes from a triangle to a linear chain. As in Fig. 2(a), three atoms, $A B C$, are initially arranged in the triangle configuration, with $\overline{A B}=\overline{B C}=d=8 \mu \mathrm{m}$, and the bending angle $\theta=$ $\angle A B C$ is gradually changed from $60^{\circ}$ (a triangle) to $180^{\circ}$ (a linear chain). The corresponding atom positions are $A(-d, 0,0), B(0,0,0)$, and $C(-d \cos \theta, d \sin \theta, 0)$ in Cartesian coordinates. The energy levels, given by the direct diagonalization of $H$, are shown in Fig. 2(b), in which the bright states $\left(\left|\lambda_{1}\right\rangle,\left|\lambda_{2}\right\rangle,\left|\lambda_{4}\right\rangle\right.$, and $\left.\left|\lambda_{5}\right\rangle\right)$ are depicted with solid lines and the dark state $\left(\left|\lambda_{3}\right\rangle\right.$, others are out of the given spectral range) with a dashed line.

There are three characteristic regimes: (i) $U_{A C}>$ $\Omega$, the superatom regime near $\theta=60^{\circ}$, (ii) $U_{A C} \sim$ $\Omega / 10$, the $A C$ double-excitation regime near $\theta=90^{\circ}$, and (iii) $U_{A C} \ll \Omega$, the linear chain regime near $\theta=$ $180^{\circ}$. In the superatom regime, (i) $U_{A C}>\Omega$, maximally one atom is excited among $A B C$, due to the blockade effect. In Fig. 2(c) we plot the time evolution of the probability, $P_{0}(t)=|\langle 000 \mid \Psi(t)\rangle|^{2}$, measured at $\theta=$ $60^{\circ}$, which shows the collective Rabi oscillation with frequency $\Omega_{c}=\sqrt{3} \Omega$. The two eigenstates, of respective energies $\lambda_{1}=-\Omega_{c} / 2$ and $\lambda_{4}=\Omega_{c} / 2$, are $\left|\lambda_{1}\right\rangle=$ $\left(\left|W_{0}\right\rangle-\left|W_{1}\right\rangle\right) / \sqrt{2}$ and $\left|\lambda_{4}\right\rangle=\left(\left|W_{0}\right\rangle+\left|W_{1}\right\rangle\right) / \sqrt{2}$, represented with symmetry base states, $\left|W_{0}\right\rangle=|000\rangle$ (the zeroexcitation state) and $\left|W_{1}\right\rangle=(|100\rangle+|010\rangle+|001\rangle) / \sqrt{3}$ (the superatom state). The Fourier transform is given in Fig. 2(d), showing the resonance agreeing with $\lambda_{4}-\lambda_{1}=$ $\Omega_{c}$ within the linewidth limited by the coherence. In regimes (ii) and (iii), the three-atom symmetry is broken. The single-excitation state $\left|W_{1}\right\rangle$ is energy split to (a)

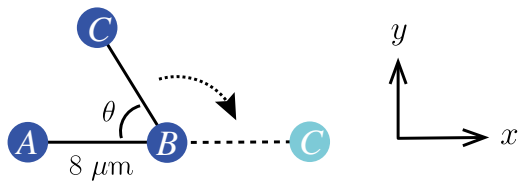

(b)
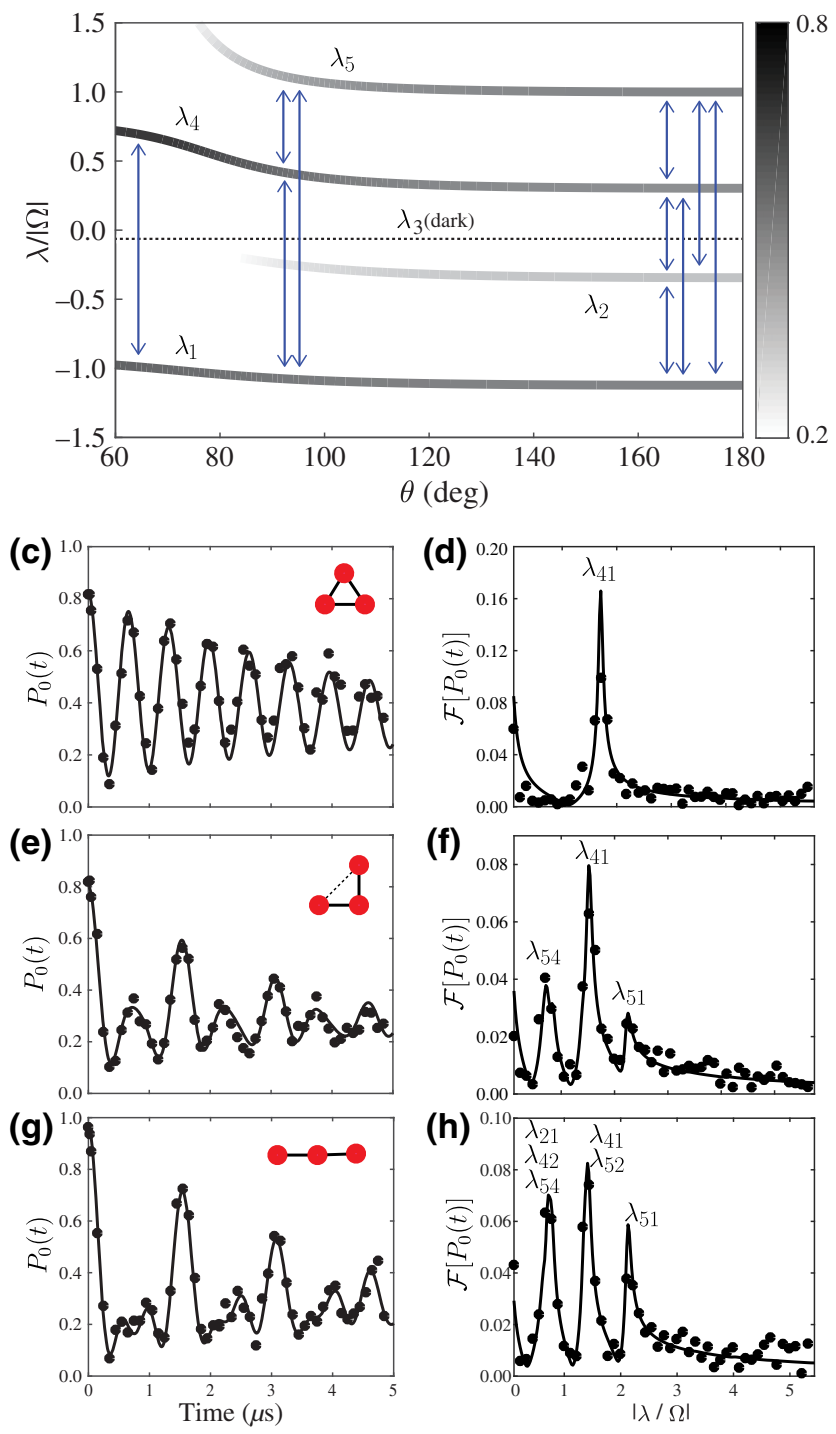

FIG. 2. (a) Configuration of three atoms, changed from a triangle $\left(\theta=60^{\circ}\right)$ to a linear chain $\left(\theta=180^{\circ}\right)$. (b) Energy levels of $H$ in Eq. (1) applied for the given three-atom configurations, in which the gray-scale solid lines represent the overlap integral $\left|\left\langle W_{0} \mid \lambda_{j}\right\rangle\right|$ of bright states and the dotted line a dark state. We respectively plot the measured time evolution of $P_{0}(t)=$ $|\langle 000 \mid \Psi(t)\rangle|^{2}$ and its Fourier transform for (c),(d) $\theta=60^{\circ}$; (e),(f) $\theta=90^{\circ}$; (g),(h) $\theta=180^{\circ}$. In (c)-(h), filled circles represent experimental data and lines represent theoretical data.

$\left|W_{1}^{\prime}\right\rangle=(|100\rangle+|001\rangle) / \sqrt{2}$ and $|010\rangle$, and the doubleexcitation state $|101\rangle$ appears. As a result, there are two additional states $\left|\lambda_{2}\right\rangle$ and $\left|\lambda_{5}\right\rangle$. The energy level calculation in Fig. 2(b) shows that the higher-energy state $\left|\lambda_{5}\right\rangle$, 
which is almost $|101\rangle$ at $\theta \sim 80^{\circ}$, starts to be coupled before $\left|\lambda_{2}\right\rangle$, indicating that the $A C$ double-excitation state $|101\rangle$ is generated in the second regime, $U_{A C} \sim \Omega / 10$. In agreement, the measurements in regime (ii), at $\theta=90^{\circ}$, are plotted in Figs. 2(e) and 2(f), showing three resonances, $\lambda_{54}, \lambda_{41}$, and $\lambda_{51}$. In regime (iii), measurements for $\theta=$ $180^{\circ}$ in Figs. $2(\mathrm{~g})$ and $2(\mathrm{~h})$ show only three resonances, while six $\left({ }_{4} C_{2}=6\right)$ are expected; however, numerical calculation of the linear chain $\left(U_{A C} \ll \Omega\right)$ confirms that the $\left|\lambda_{2}\right\rangle$ amplitude is nonzero and that two of the measured resonances are energy degenerate, $\lambda_{54}=\lambda_{42}=\lambda_{21}$ and $\lambda_{41}=\lambda_{52}$, within the spectral resolution. In Table II we summarize the eigenenergies and eigenstates of the $N=$ 3 linear chain. The lines in Figs. 2(c)-2(h) are numerical simulations of the given three-atom dynamics, carried out with the method introduced in Refs. [32,33], in which Lindbladian master equations are solved with statepreparation-and-measurement errors, intrinsic dephasing, and laser spectral noises taken into account.
TABLE II. Eigenstates of an $N=3$ linear chain with $\left|W_{0}\right\rangle=$ $|000\rangle$ and $\left|W_{1}^{\prime}\right\rangle=(|100\rangle+|001\rangle) / \sqrt{2}$.

\begin{tabular}{l}
\hline Eigenstates (bright states only) \\
$\left|\lambda_{1}=-\Omega\right\rangle=\frac{\sqrt{31}}{10}\left|W_{0}\right\rangle-\frac{\sqrt{43}}{10 \sqrt{2}}\left|W_{1}^{\prime}\right\rangle-\frac{\sqrt{7}}{10}|010\rangle+\frac{\sqrt{19}}{10}|101\rangle$ \\
$\left|\lambda_{2}=-\Omega / 3\right\rangle=\frac{\sqrt{19}}{10}\left|W_{0}\right\rangle+\frac{\sqrt{7}}{10 \sqrt{2}}\left|W_{1}^{\prime}\right\rangle-\frac{\sqrt{43}}{10}|010\rangle-\frac{\sqrt{31}}{10}|101\rangle$ \\
$\left|\lambda_{4}=\Omega / 3\right\rangle=-\frac{\sqrt{19}}{10}\left|W_{0}\right\rangle+\frac{\sqrt{7}}{10 \sqrt{2}}\left|W_{1}^{\prime}\right\rangle-\frac{\sqrt{43}}{10}|010\rangle+\frac{\sqrt{31}}{10}|101\rangle$ \\
$\left|\lambda_{5}=\Omega\right\rangle=-\frac{\sqrt{31}}{10}\left|W_{0}\right\rangle-\frac{\sqrt{43}}{10 \sqrt{2}}\left|W_{1}^{\prime}\right\rangle-\frac{\sqrt{7}}{10}|010\rangle-\frac{\sqrt{19}}{10}|101\rangle$ \\
\hline \hline
\end{tabular}

Now we probe the structural transformations of the $N=$ 4 atom system introduced in Fig. 1. Measured results for the four 4-vertex-connected graphs $S_{4}, K_{4}, C_{4}$, and $K_{4}$ $e$ are shown in Figs. 3(a)-3(b), 3(c)-3(d), 3(e)-3(f), and $3(\mathrm{~g})-3(\mathrm{~h})$, respectively. The time evolution of the $\left|W_{0}\right\rangle-$ state probability, $P_{0}(t)=|\langle 0000 \mid \Psi(t)\rangle|^{2}$, and their Fourier transforms, $\mathcal{F}\left[P_{0}(t)\right]$, are shown (filled circles) in comparison with numerical calculations (lines). Mainly four
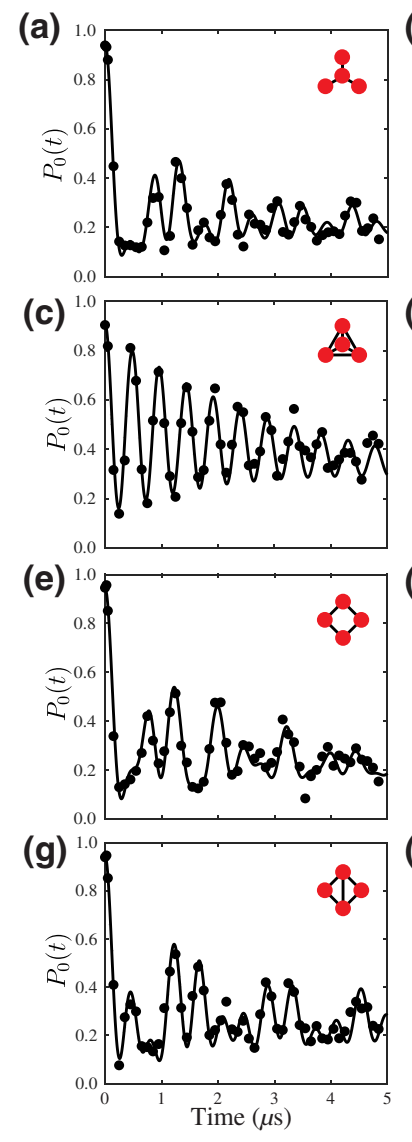

(b)

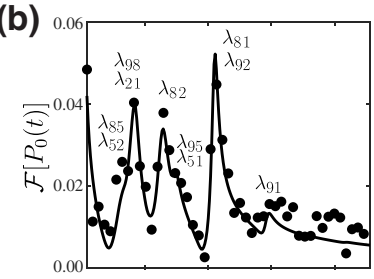

(d)

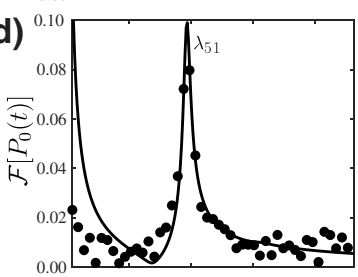

(f)

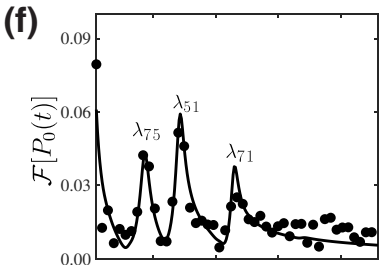

(h)

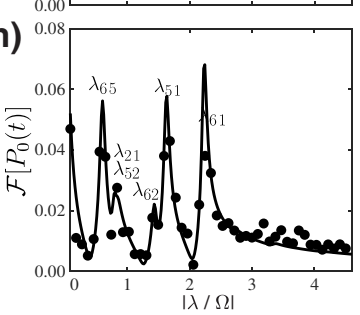

(i)
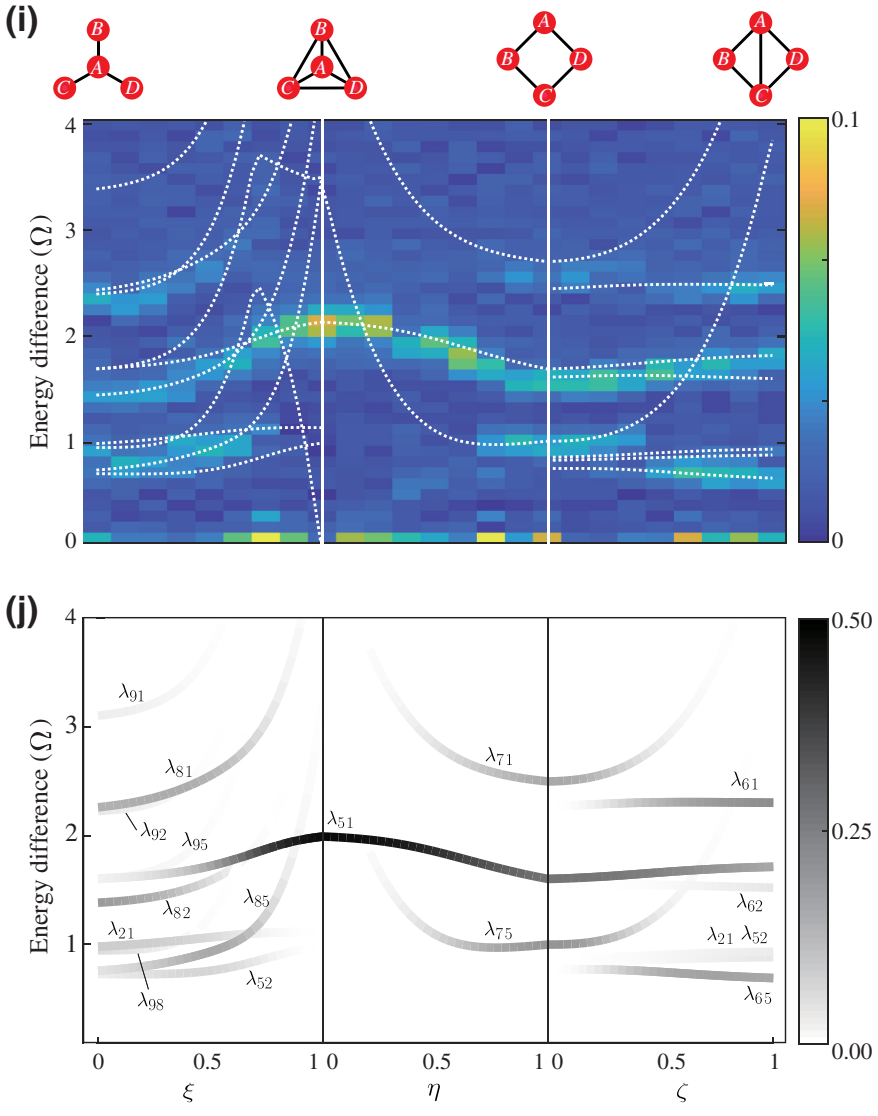

FIG. 3. Structural transformation of an $N=4$ Rydberg-atom system through the sequence $S_{4} \rightarrow K_{4} \rightarrow C_{4} \rightarrow K_{4}$-e. We respectively plot the measured $P_{0}(t)=|\langle 0000 \mid \Psi(t)\rangle|^{2}$ and Fourier transform $\mathcal{F}\left[P_{0}(t)\right]$ for (a),(b) the star graph $S_{4}$; (c), (d) the complete graph $K_{4}$; (e),(f) the cyclic graph $C_{4} ;(\mathrm{g}),(\mathrm{h})$ the diamond graph $K_{4}-e$. (i) Measured energy differences $\mathcal{F}\left[P_{0}(t)\right]$ versus the deformation parameters $\left(\xi, \eta\right.$, and $\zeta$ ). (j) Theoretical eigenenergy differences $\lambda_{j k}$ (without decoherence effects taken into account), in which the solid and dashed lines denote bright and dark transitions, respectively, and the gray scale represents the power spectral density. Note that the theoretical Fourier transforms in (b),(d),(f),(h) use a 10 times longer time window for continuous spectra, so the zero-frequency components are not physical. 
spectral peaks are observed for $S_{4}$, which correspond to the nondegenerate energy differences $\lambda_{2}-\lambda_{1}=\lambda_{9}-\lambda_{8}$, $\lambda_{8}-\lambda_{2}, \lambda_{9}-\lambda_{2}=\lambda_{8}-\lambda_{1}$, and $\lambda_{9}-\lambda_{1}$ in Table I. Likewise, $K_{4}$ has one peak, $\lambda_{5}-\lambda_{1}$, and $C_{4}$ has three peaks, $\lambda_{7}-\lambda_{5}, \lambda_{5}-\lambda_{1}$, and $\lambda_{7}-\lambda_{1}$. The diamond graph $K_{4}-e$ has three peaks, $\lambda_{6}-\lambda_{5}, \lambda_{5}-\lambda_{1}$, and $\lambda_{6}-\lambda_{1}$. We note that additional peaks are identified by numerical calculations (lines) in Figs. 3(b) and 3(h) due to the next-nearestneighbor couplings involved with $\lambda_{5}$ of $S_{4}$ and $\lambda_{2}$ of $K_{4}-e$, respectively.

Structural transformations are performed through the sequence $S_{4} \rightarrow K_{4} \rightarrow C_{4} \rightarrow K_{4}-e$, as introduced in Fig. 1 . The first transformation $S_{4} \rightarrow K_{4}$ in Fig. 1(b) is from a star to a tetrahedron, which is pulling out the center atom of $S_{4}$ from the plane of the rest three atoms, while the lengths of the three edges are maintained, until six edges of an equal length are formed. Suppose that the initial atom positions are $(0,0,0)$ and $\left(\cos \theta_{i}, \sin \theta_{i}, 0\right)$ with $\theta_{1,2,3}=0,2 \pi / 3,4 \pi / 3$. Then, the given transformation can be parameterized with the new positions $(0,0, \sqrt{2 / 3} \xi)$ and $[1-(1-2 / \sqrt{3}) \xi]\left(\cos \theta_{i}, \sin \theta_{i}, 0\right)$, where $\xi$ changes from 0 to 1 . Similarly, the second transformation $K_{4} \rightarrow C_{4}$ in Fig. 1(c) is from a tetrahedron to a square. It is stretching two nonadjacent edges of a tetrahedron, while keeping the lengths of the other four edges the same, until a square is formed. This second structural change can be defined by the new locations $[-\eta / \sqrt{2}, 0, \sqrt{2 / 3}(1-\eta)],[\sqrt{1 / 3}+(1 / \sqrt{2}-$ $\sqrt{1 / 3}) \eta, 0,0],[-(1-\eta) / 2 \sqrt{3}, 1 / 2+(1 / \sqrt{2}-1 / 2) \eta, 0]$, and $[-(1-\eta) / 2 \sqrt{3},-1 / 2+(-1 / \sqrt{2}+1 / 2) \eta, 0]$ parameterized with $\eta$ changing from $0\left(K_{4}\right)$ to $1\left(C_{4}\right)$. The last transformation $C_{4} \rightarrow K_{4}-e$ in Fig. 1(d) deforms a square to a diamond, with the parameterized locations $[\mp 1 / \sqrt{2} \mp(1 / 2-1 / \sqrt{2}) \zeta, 0,0]$ and $[0, \pm 1 / \sqrt{2} \pm$ $(\sqrt{3} / 2-1 / \sqrt{2}) \zeta, 0]$ between $\zeta=0\left(C_{4}\right)$ and $\zeta=1\left(K_{4}-\right.$ e). Experimental results are summarized in Fig. 4(i) for the given sequence of structural transformations. The Fourier transform $\mathcal{F}\left[P_{0}(t)\right]$ is plotted as a function of the parameters $\xi, \eta$, and $\zeta: \xi=0$ corresponds to $S_{4}$ in Fig. 3(b), $\eta=0$ $(\xi=1)$ to $K_{4}$ in Fig. 3(d), $\zeta=0(\eta=1)$ to $C_{4}$ in Fig. 3(f), and $\zeta=1$ to $K_{4}-e$ in Fig. 3(h). The measured spectrum is compared to the numerical calculation with $H$, the superposed dashed lines in Fig. 3(i) and the gray-scale lines in Fig. 3(j). Within the spectral resolution, the retrieved energy differences are in qualitative agreement with the numerical calculation of $H$. We note that in Fig. 3(b) additional peaks involved with $|\lambda\rangle_{5}$ are expected due to the next-nearest-neighbor interactions of the star graph, but they are not resolved in experiments.

As an additional experiment, we consider the interaction between atom clusters. As in Fig. 4, we use an $N=6$ atom system deformed from a hexagon to an antiprism (a set of (a)

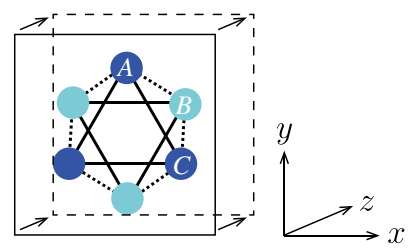

(b)

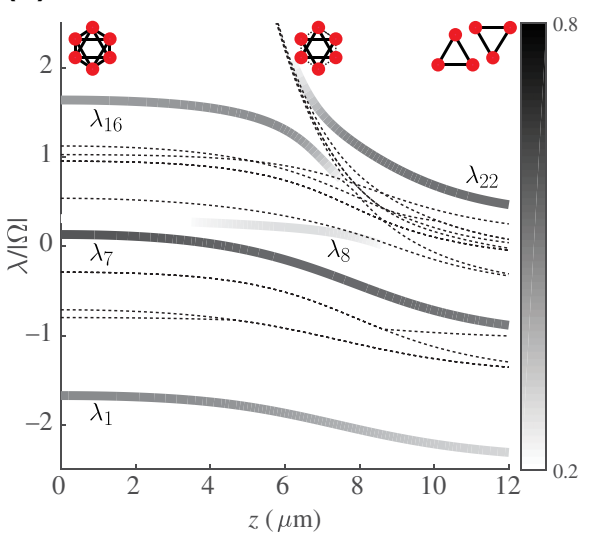

(c)

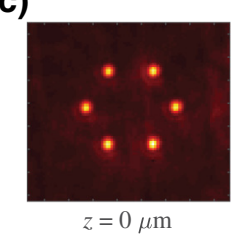

(f)

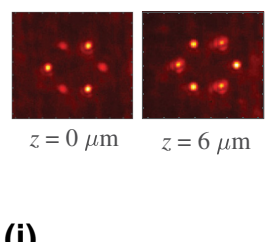

(i)

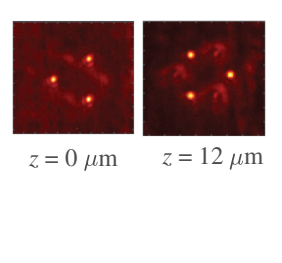

(d)

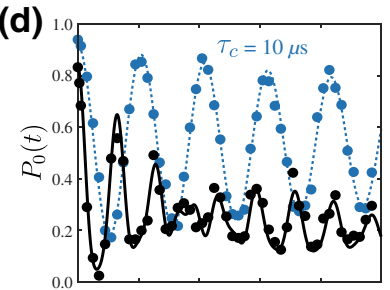

(g)

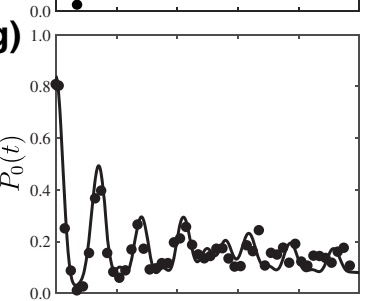

(j)

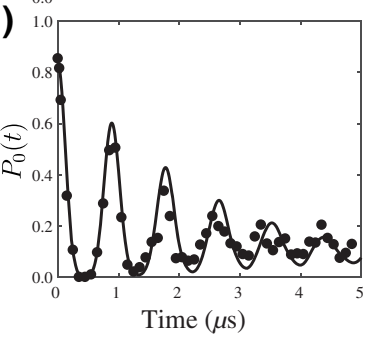

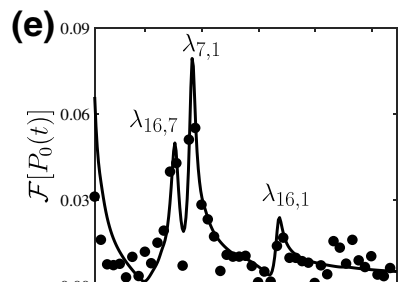

(h)

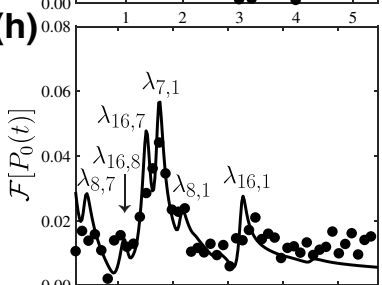

(k)

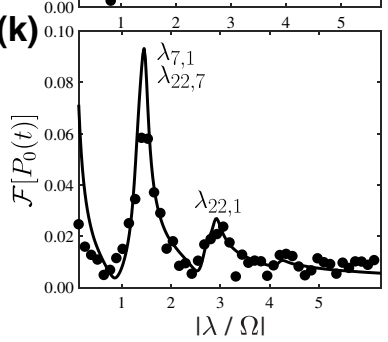

FIG. 4. Spectroscopy of $N=6$ atoms. (a) An $N=6$ atom system is deformed from a hexagon to an antiprism by axially separating two planar triangles. (b) Energy levels versus plane separation $(z)$, in which the gray scale represents the overlap integral $\left|\left\langle W_{0} \mid \lambda_{j}\right\rangle\right|$. We respectively present the fluorescence image and plots of $P_{0}(t)=\left|\left\langle W_{0} \mid \Psi(t)\right\rangle\right|^{2}$ and $\mathcal{F}\left[P_{0}(t)\right]$ for (c), (d), (e) the hexagon configuration at $z=0$; (f),(g),(h) an antiprism with $z=3 d / 2$; (i),(j),(k) an antiprism with $z=3 d / 2$. Note that blue circles in (d) are reference single-atom measurements of coherence time $\tau_{c}=10 \mu \mathrm{m}$ (see Sec. V for details). 
upright and inverted triangles, separated by $z$ ). Six atoms are initially arranged at the vertices of a hexagon, as in Fig. 4(a), with positions $d\left(\cos \theta_{i}, \sin \theta_{i}, 0\right)$ with $\theta_{j}=j \pi / 3$ (for $j=1, \ldots, 6$ ) and the axial $z$ positions of the even numbered atoms $(j=2,4,6)$ are axially translated from $z=0$ to $z=3 d / 2$. During the transformation, the length of each triangle is kept constant, $\overline{A C}=d=8 \mu \mathrm{m}$, so that the atoms in each cluster remain strongly coupled, and the distance $\overline{A B}$ is changed as $\overline{A B}(z)=\sqrt{d^{2} / 3+z^{2}}$, so that the interacting between the clusters changes. The energy levels calculated with $H$ are plotted in Fig. 4(b), which shows three distinct coupling regimes: (i) $U_{A B} \gg \Omega$, the hexagon regime at $z=0$, (ii) $U_{A B} \sim \Omega$, the $A B$ doubleexcitation regime around $z=3 d / 4$, and (iii) $U_{A B} \ll \Omega$, the decoupled trios near $z=3 d / 2$.

In the hexagon regime, (i) $U_{A B} \gg \Omega$, there are three eigenstates, constructed with symmetric base states $\left|W_{0}\right\rangle=|000000\rangle,\left|W_{1}\right\rangle$ (the superposition of singleexcitation states $)$, and $\left|W_{2}^{d}\right\rangle=(|100100\rangle+|010010\rangle+$ $|001001\rangle) / \sqrt{3}$ (the superposition of diagonal double excitations). We denote the eigenstates by $\left|\lambda_{1}\right\rangle,\left|\lambda_{7}\right\rangle$, and $\left|\lambda_{16}\right\rangle$. The results for the six atoms in the hexagon configuration at $z=0$ are shown in Figs. 4(c)-4(e) with the fluorescence image, the measured $\left|W_{0}\right\rangle$ probability, and the Fourier transform, respectively. Likewise, the results for $z=3 d / 4$ and $z=3 d / 2$ are given in Figs. 4(f) 4 (h) and 4(i) $-4(\mathrm{k})$, respectively. At $z=3 d / 4$, which we refer to as (ii) the $A B$ double-excitation regime $\left(U_{A B} \sim \Omega\right)$, the distance between $A B$ atoms is bigger than the blockade radius, i.e., $\overline{A B}>r_{b}$, so $A B$ can be excited together, while they are weakly coupled $\left(U_{A B} \sim \Omega\right)$. Therefore, besides the above base states, $\left|W_{0}\right\rangle,\left|W_{1}\right\rangle$, and $\left|W_{2}^{d}\right\rangle$, an additional symmetric base state, $\left|W_{2}^{A B}\right\rangle$ (the superposition of adjacent double excitations), is allowed. While the numerical calculation in Fig. $4(\mathrm{~h})$ predicts ${ }_{4} C_{2}=6$ peaks, the measured spectrum is little resolvable. In regime (iii), $U_{A B} \ll$ $\Omega$, at $z=3 d / 2$, the atom planes are well separated, and, as a result, the two sets of three atoms are decoupled, each constructed with its own symmetric basis, $|000\rangle$ and $(|100\rangle+|010\rangle+|001\rangle) / \sqrt{3}$. So the eigenenergies of the decoupled trios are given by $\lambda_{1}=-\sqrt{3} \Omega, \lambda_{7}=0$, and $\lambda_{22}=\sqrt{3} \Omega$.

\section{TECHNICAL DETAILS AND IMPROVEMENTS}

The spectral resolution of the given spectroscopy is mainly limited by the coherence time of the Rydberg-atom quantum simulator. It was discussed in Refs. [32,33] that the coherent operation time of the machine is dominantly limited by the nonintrinsic dephasing due to laser spectral phase noises. In order to suppress the laser spectral phase noises, we adopt the laser frequency stabilization method described in Ref. [34] without using intracavity electro-optic modulations in this work. The laser frequency is locked to a resonance of a high-finesse cavity using the
Pound-Drever-Hall (PDH) technique. The reflected light from the cavity is directed into a PDH module (Stable Laser System PDH-1000-20D) that includes a fast photodetector and electronics to demodulate the detected beat signal. The demodulated signal is fed into a fast analog proportional-integral-derivative (PID) controller (Toptica FALC 110) and two separate ("slow" and "fast") servo loops are implemented: "slow" for changing the angle of the grating in the extracavity diode laser, mostly to compensate frequency drift, and "fast" for changing the current through the laser diode, mostly to reduce the linewidth. By setting the PID parameters (to get the highest possible gain for low frequency and relatively low gain for high frequency with a proper amount of $90^{\circ}$ phase shifted signals) and optimizing the transfer function of the servo loop, we restrain the oscillation of the servo loop at the margin of the bandwidth (which otherwise caused a servo bump) and achieve the sufficiently low frequency noise level, $S_{v}(f)<10^{3} \mathrm{~Hz}^{2} / \mathrm{Hz}$, even at a servo bump around $1 \mathrm{MHz}$. With the described method, the coherence time (Rabi decay time) is measured to be $\tau_{c}=10(1) \mu \mathrm{s}$, as in Fig. 4(d), an improvement on the previous $2.5 \mu$ s [32]. The spectral resolution of the discrete Fourier transform (DFT) is given by the sampling frequency divided by the window length $\left(W_{\mathrm{DFT}}=\tau_{c} / \Delta t\right)$, so the eigenenergy resolution is $\Delta E=(1 / \Delta t) / W_{\mathrm{DFT}}=0.1 \mathrm{MHz}$ or $\Delta E=0.1 \Omega$ in our experiment.

To make the atomic arrangements of the given geometries, we extend the method of dynamic holographic optical tweezers [35], previously restricted to two-dimensional arrangements, to a three-dimensional version. The hologram on demand for an optical tweezer arrangement is calculated with a three-dimensional Gerchberg-Saxton (GS) algorithm, along with the methods of weightedGS and phase induction [35] for fast convergence. For each cycle of atom rearrangement, positions of about 26 optical tweezers are simultaneously shifted by differential displacements frame by frame, throughout a serial sequence of 35 successive phase patterns in $700 \mathrm{~ms}$, to achieve an occupation probability per site of greater than 0.94. While the transverse fluctuation of trap positions is below the imaging resolution limit, the axial position fluctuation is about $1 \mu \mathrm{m}$, due to limited phase convergence. The number of GS-algorithm iterations is set to five in the experiments, compromising between the quality of the optical tweezers and the calculation time.

A typical time budget for an experiment with a singleplane arrangement of atoms is less than one second, given by the sum of the times for atom loading $(100 \mathrm{~ms})$, initial occupancy checking $(40 \mathrm{~ms})$, atom rearrangements (700 $\mathrm{ms}$ ), final occupancy checking (40 ms), optical pumping ( $2 \mathrm{~ms})$, Rydberg-atom excitation $(5 \mu \mathrm{s})$, and final state detection $(40 \mathrm{~ms})$. When atom arrangements are repeated for a multiplane geometry, the overall time increases but 
is little significant compared to the 40-s trap life time. The signal-to-noise ratio of the given time-domain spectroscopy scales as $O\left(1 / 2^{N}\right)$, which may be improved by either resonance-enhanced probing as in coherent imaging spectroscopy [36] or a qubit-addressable quantum adiabatic process.

\section{CONCLUSION}

In summary, we utilize three-dimensional arrangements of neutral atoms, of adjustable interatom distances, to obtain the conformation energy landscape of strongly interacting, small-scale Rydberg-atom systems, in particular, during their structural transformations. We probe all possible nonisomorphic, connected graph configurations programmed for $N=3,4$ atoms and partial graphs for $N=6$. The experimentally measured graph-dependent eigenspecta are in good agreement with the model calculation of the few-body quantum Ising Hamiltonian. It is hoped that the high-dimensional programming of qubit connectivities demonstrated in this paper shall be useful for further applications of programmable quantum simulators.

\section{ACKNOWLEDGMENTS}

This research is supported by the Samsung Science and Technology Foundation (SSTF-BA1301-52), National Research Foundation of Korea (NRF) (2017R1E1A1A0 1074307), and Institute for Information \& Communications Technology Promotion (IITP-2018-2018-0-01402). We thank Woojun Lee, Hansub Hwang, and Heekun Nho for assistance in constructing three-dimensional optical tweezer traps. We also thank the anonymous referees for valuable comments.

[1] R. Feynman, Simulating physics with computers, Int. J. Theor. Phys. 21, 467 (1982).

[2] M. A. Nielsen and I. K. Chuang, Quantum Information and Quantum Computation (Cambridge University Press, Cambridge, 2000).

[3] I. M. Georgescu, S. Ashhab, and F. Nori, Quantum simulation, Rev. Mod. Phys. 86, 153 (2014).

[4] C. Gross and I. Bloch, Quantum simulations with ultracold atoms in optical lattices, Science 357, 995 (2017).

[5] L.-M. Duan and C. Monroe, Colloquium: quantum networks with trapped ions, Rev. Mod. Phys. 82, 1209 (2010).

[6] G. Wendin, Quantum information processing with superconducting circuits: A review, Rep. Prog. Phys. 80, 10 (2017).

[7] H. J. Briegel, T. Calarco, D. Jaksch, J. I. Cirac, and P. Zoller, Quantum computing with neutral atoms, J. Mod. Opt. 47, 415 (2000).

[8] D. S. Weiss and M. Saffman, Quantum computing with neutral atoms, Phys. Today 70, No. 7, 44 (2017).
[9] M. Saffman, Quantum computing with atomic qubits and Rydberg interactions: Progress and challenges, J. Phys. B: At. Mol. Opt. Phys. 49, 202001 (2016).

[10] M. D. Lukin, M. Fleischhauer, R. Cote, L. M. Duan, D. Jaksch, J. I. Cirac, and P. Zoller, Dipole Blockade and Quantum Information Processing in Mesoscopic Atomic Ensembles, Phys. Rev. Lett. 87, 037901 (2001).

[11] T. Wilk, A. Gaëtan, C. Evellin, J. Wolters, Y. Miroshnychenko, P. Grangier, and A. Browaeys, Entanglement of Two Individual Neutral Atoms Using Rydberg Blockade, Phys. Rev. Lett. 104, 010502 (2010).

[12] L. Isenhower, E. Urban, X. L. Zhang, A. T. Gill, T. Henage, T. A. Johnson, T. G. Walker, and M. Saffman, Demonstration of a Neutral Atom Controlled-NOT Quantum Gate, Phys. Rev. Lett. 104, 010503 (2010).

[13] A. Omran, H. Levine, A. Keesling, G. Semeghini, T. T. Wang, S. Ebadi, H. Bernien, A. S. Zibrov, H. Pichler, S. Choi, J. Cui, M. Rossignolo, P. Rembold, S. Montangero, T. Calarco, M. Endres, M. Greiner, V. Vuletić, and M. D. Lukin, Generation and manipulation of Schrödinger cat states in Rydberg atom arrays, Science 365, 570 (2019).

[14] M. Marcuzzi, J. Minár, D. Barredo, S. de Léséleuc, H. Labuhn, T. Lahaye, A. Browaeys, E. Levi, and I. Lesanovsky, Facilitation Dynamics and Localization Phenomena in Rydberg Lattice Gases with Position Disorder, Phys. Rev. Lett. 118, 063606 (2017).

[15] H. Kim, Y. J. Park, K. Kim, H. S. Sim, and J. Ahn, Detailed Balance of Thermalization Dynamics in RydbergAtom Quantum Simulators, Phys. Rev. Lett. 120, 180502 (2018).

[16] H. Labuhn, D. Barredo, S. Ravets, S. de Léséleuc, T. Macrì, and T. Lahaye, Tunable two-dimensional arrays of single Rydberg atoms for realizing quantum Ising models, Nature 534, 667 (2016).

[17] V. Lienhard, S. de Léséleuc, D. Barredo, T. Lahaye, A. Browaeys, M. Schuler, L.-P. Henry, and A. M. Läuchli, Observing the Space- and Time-Dependent Growth of Correlations in Dynamically Tuned Synthetic Ising Models with Antiferromagnetic Interactions, Phys. Rev. X 8, 021070 (2018).

[18] R. Samajdar, W. W. Ho, H. Pichler, M. D. Lukin, and S. Sachdev, Complex Density Wave Orders and Quantum Phase Transitions in a Model of Square-Lattice Rydberg Atom Arrays, Phys. Rev. Lett. 124, 103601 (2020).

[19] D. Barredo, V. Lienhard, S. de Léséleuc, T. Lahaye, and A. Browaeys, Synthetic three-dimensional atomic structures assembled atom by atom, Nature 561, 79 (2018).

[20] A. Browaeys and T. Lahaye, Many-body physics with individually controlled Rydberg atoms, Nat. Phys. 16, 132 (2020).

[21] H. Kim, W. Lee, H.-G. Lee, H. Jo, Y. Song, and J. Ahn, In situ single-atom array synthesis by dynamic holographic optical tweezers, Nat. Commun. 7, 13317 (2016).

[22] D. Barredo, S. de Léséleuc, V. Lienhard, T. Lahaye, and A. Browaeys, An atom-by-atom assembler of defect-free arbitrary 2D atomic arrays, Science 354, 1021 (2016).

[23] M. Endres, H. Bernien, A. Keesling, H. Levine, E. R. Anschuetz, A. Krajenbrink, and M. D. Lukin, Atom-byatom assembly of defect-free one-dimensional cold atom arrays, Science 354, 1024 (2016). 
[24] S. de Léséleuc, V. Lienhard, P. Scholl, D. Barredo, S. Weber, N. Lang, H. P. Büchler, T. Lahaye, and A. Browaeys, Observation of a symmetry protected topological phase of interacting bosons with Rydberg atoms, Science 365, 775 (2019).

[25] A. Keesling, A. Omran, H. Levine, H. Bernien, H. Pichler, S. Choi, R. Samajdar, S. Schwartz, P. Silvi, S. Sachdev, P. Zoller, M. Endres, M. Greiner, V. Vuletić, and M. D. Lukin, Quantum Kibble-Zurek mechanism and critical dynamics on a programmable Rydberg simulator, Nature 568, 207 (2019).

[26] H. Bernien, S. Schwartz, A. Keesling, H. Levine, A. Omran, H. Pichler, S. Choi, A. S. Zibrov, M. Endres, M. Greiner, V. Vuletić, and M. D. Lukin, Probing many-body dynamics on a 51-atom quantum simulator, Nature 551, 579 (2016).

[27] F. Arute, et al., Quantum supremacy using a programmable superconducting processor, Nature 574, 505 (2019).

[28] H. Pichler, S.-T. Wang, L. Zhou, S. Choi, and M. D. Lukin, arXiv: 1808.10816.

[29] W. Lee, H. Kim, and J. Ahn, Three-dimensional rearrangement of single atoms using actively controlled optical microtraps, Opt. Express 24, 9816 (2016).
[30] W. Lee, H. Kim, and J. Ahn, Defect-free atomic array formation using the Hungarian matching algorithm, Phys. Rev. A 95, 053424 (2017).

[31] H. Jo, Y. Song, M. Kim, and J. Ahn, Rydberg Atom Entanglements in the Weak Coupling Regime, Phys. Rev. Lett. 124, 033603 (2020).

[32] W. Lee, M. Kim, H. Jo, Y. Song, and J. Ahn, Coherent and dissipative dynamics of entangled few-body systems of Rydberg atoms, Phys. Rev. A 99, 043404 (2019).

[33] H. Tamura, T. Yamakoshi, and K. Nakagawa, Analysis of coherent dynamics of a Rydberg-atom quantum simulator, Phys. Rev. A 101, 043421 (2020).

[34] J. Le Gouët, J. Kim, C. Bourassin-Bouchet, M. Lours, A. Landragin, and F. Pereira Dos Santos, Wide bandwidth phase-locked diode laser with an intra-cavity electro-optic modulator, Opt. Commun. 282, 977 (2009).

[35] H. Kim, M. Kim, W. Lee, and J. Ahn, GerchbergSaxton algorithm for tweezer-trap atom arrangements, Opt. Express 27, 2184 (2019).

[36] C. Senko, J. Smith, P. Richerme, A. Lee, W. C. Campbell, and C. Monroe, Coherent imaging spectroscopy of a quantum many-body spin system, Science 345, 430 (2014) 